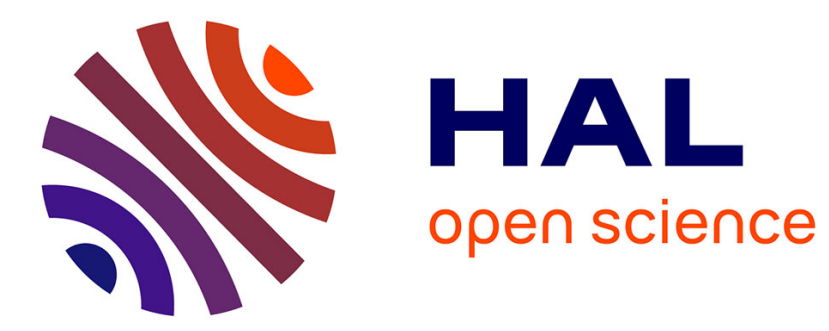

\title{
Three-dimensional vibrations of Tethered Satellite System
}

\author{
Monica Pasca, Marcello Pignataro, Angelo Luongo
}

\section{To cite this version:}

Monica Pasca, Marcello Pignataro, Angelo Luongo. Three-dimensional vibrations of Tethered Satellite System. Journal Control and Guidance, 1991, 14 (2), pp.312-320. hal-00790022

\section{HAL Id: hal-00790022 \\ https://hal.science/hal-00790022}

Submitted on 19 Feb 2013

HAL is a multi-disciplinary open access archive for the deposit and dissemination of scientific research documents, whether they are published or not. The documents may come from teaching and research institutions in France or abroad, or from public or private research centers.
L'archive ouverte pluridisciplinaire HAL, est destinée au dépôt et à la diffusion de documents scientifiques de niveau recherche, publiés ou non, émanant des établissements d'enseignement et de recherche français ou étrangers, des laboratoires publics ou privés. 


\title{
Three-Dimensional Vibrations of Tethered Satellite Systems
}

\author{
Monica Pasca* and Marcello Pignataro $\dagger$ \\ University of Rome, Rome, Italy \\ and \\ Angelo Luongo \\ University of L'Aquila, L'Aquila, Italy
}

\begin{abstract}
The three-dimensional free vibrations of an orbiter-towed tethered satellite system with nominally vertical tether during stationkeeping phase are studied analytically. The tether is modeled as an elastic continuum with mass and the orbiter and the subsatellite as two concentrated masses. The linearized equations of motion with variable coefficients are solved by means of a perturbation technique. An analysis of the order of magnitude of the different terms involved permits evaluation of the coupling between longitudinal and transversal in-plane vibrations coming to an approximate closed-form solution of the eigenvalue problem.
\end{abstract}

\section{Introduction}

$\mathbf{T}$ HE vast potential of the tethered satellite system (TSS) ${ }^{1,2}$ within the space research program has recently stimulated many investigations on its dynamic behavior and control. Most of the work deals with deployment and retrieval of the subsatellite and the determination of the most appropriate control law. ${ }^{3-5}$ Yet despite its importance, little attention has been devoted to the dynamics of the stationkeeping phase. ${ }^{6-10}$ Knowledge of the elastic oscillations of the system is therefore necessary to evaluate the disturbances introduced into the experimental observations. A survey on the development in these areas has been provided by Misra and Modi. ${ }^{11}$

A continuum model was formulated previously ${ }^{12}$ by the authors in order to investigate the free dynamics of the TSS during the stationkeeping phase. The following simplifying assumptions were introduced: 1) the orbiter and the satellite are considered as two concentrated masses, 2) the tether is modeled as an elastic continuum with mass, and 3) the orbital eccentricity and the aerodynamic drag forces are neglected so that the tether can be assumed vertical. The nonlinear equations of motion have been derived through an energy method and then linearized around the static configuration. Out-ofplane oscillations have been studied separately because the relevant linear equations are uncoupled from in-plane equations of motion.

In this paper, by using the model previously introduced, the in-plane motion is analyzed. The governing equations in the longitudinal and transversal components are coupled by means of gyroscopic forces, leading to a nonstandard eigenvalue problem. ${ }^{13,14} \mathrm{It}$ is found that the eigenvalue problem in the real and imaginary parts of the eigenfunctions of the gyroscopic system splits into two equal problems because of the particular structure of the mass and stiffness operators. A perturbation technique is used to solve the system characterized by variable coefficients arising from the stress gradient along the tether. As a result, eigenvalues and eigenfunctions are determined by perturbing those relative to a constant state of stress. In order to evaluate the influence of different terms in the equations of motion, an asymptotic analysis has been

\footnotetext{
*Doctorate Student, Department of Structural and Geotechnical Engineering.

$\nmid$ Professor, Department of Structural and Geotechnical Engineering.

$\$$ Professor, Department of Structural Engineering.
}

performed. This shows that, within technical values of the geometric and mechanical parameters, the two components of motion are weakly coupled by gyroscopic forces and two sets of eigenfunctions exist, one prevalently longitudinal and the other prevalently transversal. These conclusions are supported by numerical results.

In the authors' opinion, some new contributions have been made with respect to previous papers, arising from 1) the use of a more sophisticated model and 2) the use of perturbation techniques in computing eigenfrequencies and eigenvectors. In particular, the gyroscopic forces associated with the in-plane motion are taken into account and no assumptions are introduced on the value of the satellite/orbiter mass ratio. As a consequence, the model permits a more accurate description of the motion and enables the systems to be analyzed with lumped masses of the same order. Furthermore, the perturbation method employed permits attainment of a simple closedform solution, in comparison, for example, with Ref. 10, although it cannot always be applied. Finally, the asymptotic analysis based on the estimate of the order of magnitude of the terms of the equations of motion provides important insights into the mechanical behavior of the system.

\section{Formulation of the Problem}

Let us consider the system consisting of a Space Shuttle (SS) and a subsatellite, connected by an elastic tether. Let ( $O, X$, $Y, Z)$ be a rest frame whose origin coincides with the Earth mass center. Assume that the SS describes a circular, undisturbed orbit of radius $a$ in the $X-Y$ plane, with constant angular velocity $n$. It is convenient to introduce a reference system $(o, x, y, z)$ whose origin coincides with the SS center of mass; the $x, y$ axes lie in the $X-Y$ plane and are oriented as shown in Fig. 1. A curvilinear abscissa $S$ measured from the SS along the tether is also introduced.

Accounting for the kinetic and potential energies, the Lagrangian of the system is

$$
\begin{aligned}
L & =\frac{1}{2}\left\{\mu \int_{0}^{\ell}|\dot{r}+n \times \boldsymbol{r}|^{2} \mathrm{~d} S+m_{1}|\dot{r}+n \times r|_{S=0}^{2}\right. \\
& \left.+m_{2}|\dot{r}+\boldsymbol{n} \times \boldsymbol{r}|_{S=\ell}^{2}\right\} \\
& +\mu_{e}\left\{\mu \int_{0}^{\ell} \mathrm{d} S /|\boldsymbol{r}|+m_{1} /|\boldsymbol{r}|_{S=0}+m_{2} /|\boldsymbol{r}|_{S=\ell}\right\} \\
& -\frac{1}{2} E A \int_{0}^{\ell} e^{2} \mathrm{~d} S
\end{aligned}
$$




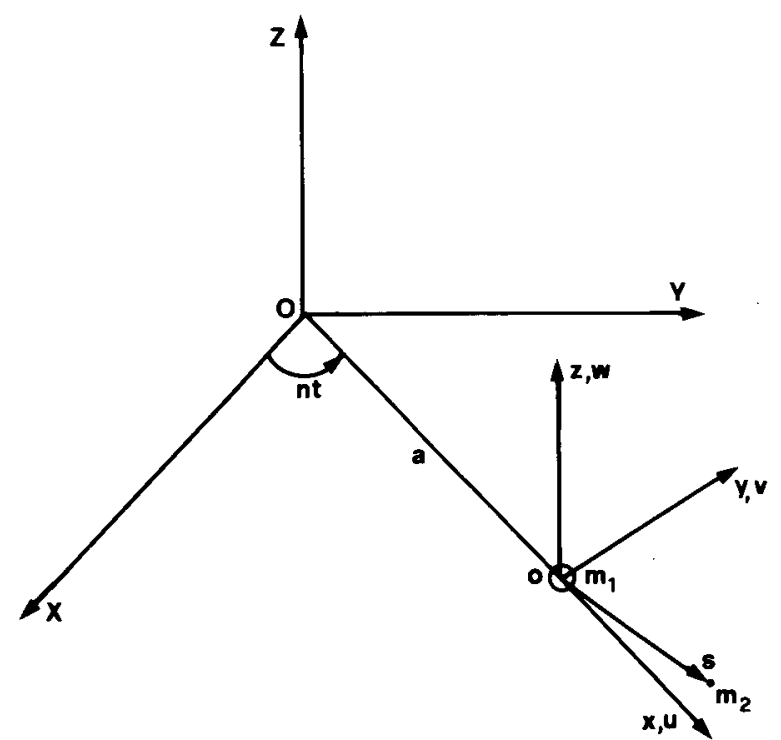

Fig. 1 TSS model.

where a dot indicates differentiation with respect to time $\bar{t}$. In Eq. (1), $\mu_{e}$ denotes the gravitational constant, $\mu$ the mass density of the tether, $\ell$ its unstretched length, $A$ its cross section, $E$ the Young's modulus, and $m_{1}$ and $m_{2}$ the masses of the SS and the subsatellite, respectively. In addition, $r$ is the position vector, $r=(a+x) c_{1}+y c_{2}+z c_{3}$, where $c_{1} c_{2} c_{3}$ are the unit vectors of the moving system, and $e$ is the stretching defined as $e=\left[\left(x^{\prime 2}+y^{\prime 2}+z^{\prime 2}\right)^{1 / 2}-1\right]$, where a prime denotes differentiation with respect to $S$.

From the stationary condition of the Lagrangian, the general equations of motion are obtained

$$
\begin{aligned}
& \mu \ddot{x}-2 \mu n \dot{y}-\mu n^{2}(a+x) \\
& +\mu \mu_{e} \frac{(a+x)}{|r|^{3}}-E A\left[x^{\prime} \frac{e}{(1+e)}\right]^{\prime}=0 \\
& \left.\mu \ddot{y}+2 \mu n \dot{x}-\mu n^{2} y+\mu \mu_{e} \frac{y}{|r|^{3}}\right]^{\prime}=0 \\
& -E A\left[y^{\prime} \frac{e}{(1+e)}\right]^{\prime}=0 \\
& \mu \ddot{z}+\mu \mu_{e} \frac{z}{|\boldsymbol{r}|^{3}}-E A\left[z^{\prime} \frac{e}{(1+e)}\right]^{\prime}=0
\end{aligned}
$$

with appropriate boundary conditions.

Equations (2) admit a steady-state solution $x=x(S), y=0$, $z=0$, where $x(S)$ is obtained by solving the nonlinear equation

$$
-\mu n^{2}(a+x)+\frac{\mu \mu_{e}}{(a+x)^{2}}-E A x^{\prime \prime}=0
$$

with relevant boundary conditions.

By considering the motion with respect to the static configuration and linearizing in the additional displacement components $U, V, W$, along $x, y, z$ axes, respectively, we get the nondimensional equations of motion

$$
\begin{gathered}
\alpha^{2} \ddot{u}-2 \alpha^{2} \dot{v}-3 \alpha^{2} u-u^{\prime \prime}=0 \\
\alpha^{2} \ddot{v}+2 \alpha^{2} \dot{u}-\alpha^{2}\left[f(s) v^{\prime}\right]^{\prime}=0 \\
\alpha^{2} \ddot{w}+\alpha^{2} w-\alpha^{2}\left[f(s) w^{\prime}\right]^{\prime}=0
\end{gathered}
$$

with the associated boundary conditions

$$
\begin{aligned}
& {\left[\alpha^{2} \ddot{u}-2 \alpha^{2} \dot{v}-3 \alpha^{2} u-\beta u^{\prime}\right]_{s=0}=0} \\
& {\left[\alpha^{2} \ddot{u}-2 \alpha^{2} \dot{v}-3 \alpha^{2} u+\gamma u^{\prime}\right]_{s=1}=0} \\
& {\left[\alpha^{2} \ddot{v}+2 \alpha^{2} \dot{u}-\alpha^{2} \beta f(s) v^{\prime}\right]_{s=0}=0} \\
& {\left[\alpha^{2} \ddot{v}+2 \alpha^{2} \dot{u}+\alpha^{2} \gamma f(s) v^{\prime}\right]_{s=1}=0} \\
& {\left[\alpha^{2} \ddot{w}+\alpha^{2} w-\alpha^{2} \beta f(s) w^{\prime}\right]_{s=0}=0} \\
& {\left[\alpha^{2} \ddot{w}+\alpha^{2} w+\alpha^{2} \gamma f(s) w^{\prime}\right]_{s=1}=0}
\end{aligned}
$$

where $u=U / \ell, v=V / \ell, w=W / \ell$, and a prime and a dot denote differentiations with respect to $s=S / \ell$ and $t=\bar{t} / n$. The following nondimensional parameters have also been introduced

$$
\alpha^{2}=\frac{\mu n^{2} l^{2}}{E A}, \quad \beta=\frac{\mu l}{m_{1}}, \quad \gamma=\frac{\mu l}{m_{2}}
$$

with the function

$$
f(s)=\frac{1}{\alpha^{2}} \frac{x^{\prime}-1}{x^{\prime}}
$$

which is related to the axial stress by $N(s)=\mu n^{2} \ell^{2} f(s) x^{\prime}(s)$.

An approximate form for $f(s)$ is obtained by linearizing Eq. (3) and neglecting the stretching of the tether. The following expression is obtained

$$
f(s)=3\left[\frac{\delta}{\beta}+\left(s \delta-\frac{s^{2}}{2}\right)\right]
$$

where $\delta=d / \ell$ is the nondimensional distance of the system center of mass from the SS. As a particular case, for $\beta \rightarrow 0$ is $\delta \rightarrow 0, \delta / \beta \rightarrow 1 / 2+1 / \gamma$.

Equations (4) show that out-of-plane and in-plane motions are uncoupled and can be solved separately.

\section{In-Plane Vibrations}

\section{Solution Procedure}

By using matrix notation, Eqs. (4a) and (4b) with corresponding boundary conditions, Eqs. (5a-5d), can be written in the form

$$
M \ddot{q}+G \dot{q}+K q=0
$$

where $q(s, t)$ is the generalized coordinates vector

$$
q(s, t)=\{u(s, t), u(0, t), u(1, t), v(s, t), v(0, t), v(1, t)\}^{T}
$$

The operators $\boldsymbol{M}, \boldsymbol{G}$, and $\boldsymbol{K}$ are given by

$$
\begin{gathered}
\boldsymbol{M}=\left[\begin{array}{cc}
m & 0 \\
0 & m
\end{array}\right], \quad \boldsymbol{G}=2\left[\begin{array}{cc}
0 & -m \\
m & 0
\end{array}\right] \\
\boldsymbol{K}=\left[\begin{array}{cc}
\boldsymbol{K}_{u} & 0 \\
0 & \boldsymbol{K}_{v}
\end{array}\right]
\end{gathered}
$$

where

$$
\begin{gathered}
m=\operatorname{diag}\left(1, \frac{1}{\beta}, \frac{1}{\gamma}\right) \\
K_{u}=-\operatorname{diag}\left(3+\frac{1}{\alpha^{2}} \frac{\mathrm{d}^{2}}{\mathrm{~d} s^{2}}, \frac{3}{\beta}+\left.\frac{1}{\alpha^{2}} \frac{\mathrm{d}}{\mathrm{d} s}\right|_{s=0}\right. \\
\left.\frac{3}{\gamma}-\left.\frac{1}{\alpha^{2}} \frac{\mathrm{d}}{\mathrm{d} s}\right|_{s=1}\right)
\end{gathered}
$$




$$
\boldsymbol{K}_{v}=-\operatorname{diag}\left(\left[f \frac{\mathrm{d}}{\mathrm{d} s}\right]^{\prime},\left.f \frac{\mathrm{d}}{\mathrm{d} s}\right|_{s=0},-\left.f \frac{\mathrm{d}}{\mathrm{d} s}\right|_{s=1}\right)
$$

The linear equation [Eq. (9)] admits a solution of the form $\boldsymbol{q}(s, t)=\boldsymbol{q}(s) e^{i \omega t}$, which, after separation of variables, leads to

$$
-\omega^{2} \boldsymbol{M q}+i \omega \boldsymbol{G} \boldsymbol{q}+\boldsymbol{K} \boldsymbol{q}=\mathbf{0}
$$

Since $G$ is real, $q(s)$ in Eq. (13) must be complex in order to have a nontrivial solution. On account of the particular structure of the matrices involved, it is convenient to partition $q(s)$ into $q=(u, v)^{T}$, where $u=u_{r}+i u_{i}$ and $v=v_{r}+i v_{i}$. Equation (13) then may be rewritten as four equations,

$$
\begin{gathered}
\omega^{2} m u_{r}-2 \omega m v_{i}-K_{u} u_{r}=0 \\
\omega^{2} m v_{i}-2 \omega m u_{r}-K_{v} v_{i}=0 \\
\omega^{2} m u_{i}+2 \omega m v_{r}-K_{u} u_{i}=0 \\
\omega^{2} m v_{r}+2 \omega m u_{i}-K_{v} v_{r}=0
\end{gathered}
$$

where 12 variables are present. It is apparent that Eqs. (14) are coupled two by two and that the two systems of equations are equal if we pose $u_{i}=u_{r}, v_{r}=-v_{i}$. By denoting with $z$ the vector $z=\left(u_{r}, v_{i}\right\}^{T}$, we can rewrite Eq. (13) as

$$
\omega^{2} M z-\omega G^{*} z-K z=0
$$

where $\boldsymbol{M}$ and $\boldsymbol{K}$ are the same as before, whereas $G^{*}$ is now a symmetric matrix defined as

$$
G^{*}=2\left[\begin{array}{cc}
0 & m \\
m & 0
\end{array}\right]
$$

After solving Eq. (15), we find that the general solution for the displacement components is given by

$$
\begin{gathered}
u(s, t)=\Sigma_{j} u_{r j}(s) C_{j} \sin \left(\omega_{j} t+\psi_{j}\right) \\
v(s, t)=\Sigma_{j} v_{i j}(s) C_{j} \cos \left(\omega_{j} t+\psi_{j}\right)
\end{gathered}
$$

where $C_{j}$ and $\psi_{j}$ are arbitrary constants to be determined from initial conditions.

The frequencies of the system can be related to the eigenfunctions $\boldsymbol{u}_{r}$ and $\boldsymbol{v}_{i}$ by the following expression

$$
\omega^{2}=\frac{\int_{0}^{1} v_{i}^{T} K_{\nu} v_{i} \mathrm{~d} s-\int_{0}^{1} u_{r}^{T} K_{u} u_{r} \mathrm{~d} s}{\int_{0}^{1} v_{i}^{T} m v_{i} \mathrm{~d} s-\int_{0}^{1} u_{r}^{T} \boldsymbol{m} u_{r} \mathrm{~d} s}
$$

in which the single terms represent the contribution to potential and kinetic energy of each component of motion. In the following, subscripts $r$ and $i$ will be omitted.

A perturbation technique is now employed to solve the differential equations with variable coefficients [Eq. (15)]. To this end, in the hypothesis $\gamma \leq \mathcal{O}(1), \beta / \gamma \leq \mathcal{O}(1)$, it is possible to consider the function $f(s)$ as the sum of a constant and a variable term

$$
f(s)=f_{0}+\epsilon f_{1}(s)
$$

with $\epsilon \ll 1$. For example, by using Eq. (8), it is possible to assume $f_{0}=f(1)=f_{\min }$ (if $\left.m_{2}<m_{1}\right)$, or $f_{0}=f(\delta)=f_{\max }$, or $f_{0}$ $=f_{\mathrm{av}}=3(\delta / \beta+\delta / 2-1 / 6)$. The influence of the choice of $f_{0}$ will be discussed with numerical results.

According to Eq. (19), $K(s)$ can be written as

$$
\boldsymbol{K}(s)=\boldsymbol{K}_{0}+\epsilon \boldsymbol{K}_{1}(s)=\left[\begin{array}{cc}
\boldsymbol{K}_{u} & \mathbf{0} \\
\mathbf{0} & \boldsymbol{K}_{0 v}
\end{array}\right]+\epsilon\left[\begin{array}{cc}
\mathbf{0} & \mathbf{0} \\
\mathbf{0} & \boldsymbol{K}_{1 v}
\end{array}\right]
$$

where

$$
\begin{gathered}
\boldsymbol{K}_{0 v}=-\operatorname{diag}\left(f_{0} \frac{\mathrm{d}^{2}}{\mathrm{~d} s^{2}},\left.f_{0} \frac{\mathrm{d}}{\mathrm{d} s}\right|_{s=0},-\left.f_{0} \frac{\mathrm{d}}{\mathrm{d} s}\right|_{s=1}\right) \\
\boldsymbol{K}_{1 v}=-\operatorname{diag}\left(\left[f_{1} \frac{\mathrm{d}}{\mathrm{d} s}\right]^{\prime},\left[f_{1} \frac{\mathrm{d}}{\mathrm{d} s}\right]_{s=0},-\left[f_{1} \frac{\mathrm{d}}{\mathrm{d} s}\right]_{s=1}\right)
\end{gathered}
$$

and $K_{u}$ is defined by Eq. (12b). Expanding $z(s)$ and $\omega$ in terms of $\epsilon$ using

$$
\begin{gathered}
z(s)=z_{0}+\epsilon z_{1}+\mathcal{O}\left(\epsilon^{2}\right) \\
\omega=\omega_{0}+\epsilon \omega_{1}+\mathcal{O}\left(\epsilon^{2}\right)
\end{gathered}
$$

we obtain the perturbation equations at the order $\epsilon^{0}$ and $\epsilon$, respectively

$$
\begin{gathered}
\omega_{0}^{2} \boldsymbol{M} z_{0}-\omega_{0} G^{*} z_{0}-K_{0} z_{0}=0 \\
\omega_{0}^{2} M z_{1}-\omega_{0} G^{*} z_{1}-K_{0} z_{1}=-2 \omega_{0} \omega_{1} M z_{0}+\omega_{1} G^{*} z_{0}+K_{1} z_{0}
\end{gathered}
$$

At the zeroth order, the equation describes the problem of the TSS with constant stress along the tether. Although the associated eigenvalue problem is not a standard one, particular properties of the eigensolutions can be found. From Eq. (23), written for two different eigenvalues with the corresponding eigenfunctions, performing straightforward manipulations, we get the orthonormalization condition

$$
\left(\omega_{0 k}+\omega_{0 h}\right) \int_{0}^{1} z_{0 k}^{T} M z_{0 h} \mathrm{~d} s-\int_{0}^{1} z_{0 k}^{T} G^{*} z_{0 h} \mathrm{~d} s=\delta_{h k}
$$

The first-order solution can be determined from Eq. (24) by writing $z_{1 j}$ as a linear combination of the eigenfunctions $z_{0}$

$$
z_{1 j}=\Sigma_{k} a_{j k} z_{0 k}
$$

By applying a standard procedure, we obtain the correction to the frequency $\omega_{0 j}$,

$$
\omega_{1 j}=\int_{0}^{1} z_{0 j}^{T} K_{1} z_{0 j} \mathrm{~d} s
$$

where the eigenfunctions $z_{0 j}$ have been normalized according to Eq. (25). The coefficients $a_{j k}$ are given by the solution of a linear algebraic coupled system (see Ref. 15).

\section{Explicit Solution of the Eigenvalue Problem}

The explicit solution of Eqs. (23) and (24) implies two steps, the first corresponding to the problem with constant stress along the tether (zeroth-order perturbation solution), the second one furnishing the corrections due to the variability of the tension (first-order perturbation solution).

The zeroth-order perturbation, Eq. (23), admits the solution

$$
z_{0}(s)=Z e^{\vartheta s}
$$

By introducing Eq. (28) into Eq. (23), we obtain a fourthdegree characteristic equation whose solutions are

$$
\vartheta^{2}=-\frac{1}{2}\left(3 \alpha^{2}+\omega_{0}^{2} \alpha^{2}+\frac{\omega_{0}^{2}}{f_{0}}\right)(1 \pm \sqrt{1+\zeta})
$$

where

$$
\zeta=\frac{4 \omega_{0}^{2} \alpha^{2}\left(1-\omega_{0}^{2}\right)}{f_{0}\left(3 \alpha^{2}+\omega_{0}^{2} \alpha^{2}+\frac{\omega_{0}^{2}}{f_{0}}\right)^{2}}
$$


It is possible to show that $(1+\zeta)$ is positive for any value of $\omega_{0}$ as it can be written as the sum of two squares. Furthermore, the following inequalities hold $0>\zeta>-1$ for $\omega_{0}^{2}>1, \zeta=0$ for $\omega_{0}^{2}=1$, and $\zeta>0$ for $1>\omega_{0}^{2}>0$; consequently the roots can be real or imaginary. Only the case $\omega_{0}>1$ is analyzed here since $\omega_{0} \leq 1$ corresponds to values of the geometric and mechanical parameters, which are out of the ranges of technical interest (see Ref. 15). By defining

$$
\begin{aligned}
& p^{2}=\frac{\alpha^{2}}{2}\left[3+\omega_{0}^{2}+\frac{\omega_{0}^{2}}{\alpha^{2} f_{0}}\right](1+\sqrt{1+\zeta}) \\
& q^{2}=\frac{\alpha^{2}}{2}\left[3+\omega_{0}^{2}+\frac{\omega_{0}^{2}}{\alpha^{2} f_{0}}\right](1-\sqrt{1+\zeta})
\end{aligned}
$$

we can write the roots $\vartheta$ as $\pm i p$ and $\pm i q$ and the displacement components read

$$
u_{0}(s)=c_{p}\left(A_{1} \sin p s+A_{2} \cos p s\right)+c_{q}\left(A_{3} \sin q s+A_{4} \cos q s\right)
$$

$$
v_{0}(s)=A_{1} \sin p s+A_{2} \cos p s+A_{3} \sin q s+A_{4} \cos q s
$$

where

$$
\begin{gathered}
c_{p}=\frac{2 \omega_{0}}{3+\omega_{0}^{2}-p^{2} / \alpha^{2}} \\
c_{q}=\frac{2 \omega_{0}}{3+\omega_{0}^{2}-q^{2} / \alpha^{2}}
\end{gathered}
$$

By imposing boundary conditions, we get a homogeneous system of linear algebraic equations in the unknown amplitudes $A_{i}(i=1,2,3,4)$. The vanishing of the determinant of the coefficients leads to a transcendental characteristic equation in the unknown $\omega_{0}$, which has to be numerically solved. From this, $p$ and $q$ and, therefore, the solutions $u_{0}(s)$ and $v_{0}(s)$ are determined.

The first-order corrections to the frequencies are evaluated easily by means of Eq. (27), which, in this specific case, reads

$$
\omega_{1 j}=\int_{0}^{1} f_{1}(s) v_{0 j}^{\prime 2} \mathrm{~d} s
$$

where $v_{0 j}(s)$ is furnished by Eq. (32b).

The eigenfunctions' corrections are determined by numerically solving the system for coefficients $a_{j k}$ (see Ref. 15) where equations are coupled, since eigenfunctions are not orthogonal in the classical sense defined for natural systems, i.e., systems with no gyroscopic forces.

\section{Out-of-Plane Vibrations}

The out-of-plane motion has been studied in Ref. 12; the relevant results are summarized here. The problem is governed by $\mathrm{Eq}$. (4c) with the boundary conditions of Eqs. (5e) and (5f). By posing $w(s, t)=w(s) e^{i \omega t}$, we can write them in the matrix form

$$
\left(1-\Omega^{2}\right) \boldsymbol{M w}+\boldsymbol{K} \boldsymbol{w}=\mathbf{0}
$$

where $\boldsymbol{M}=\boldsymbol{m}, \boldsymbol{K}=\boldsymbol{K}_{v}$, as defined previously for the in-plane motion, and $w=\{w(s), w(0), w(1)\}^{T}$. The solution of the problem is obtained through the perturbation procedure previously employed by expanding $\Omega^{2}$ and $w$ into series of the small parameter $\epsilon$ appearing in Eq. (19). The relevant perturbation equations are

$$
\left(\Omega_{0}^{2}-1\right) M w_{0}-K_{0} w_{0}=0
$$

$$
\left(\Omega_{0}^{2}-1\right) M w_{1}-K_{0} w_{1}=-\Omega_{1}^{2} M w_{0}+K_{1} w_{0}
$$

where $K_{0}$ and $K_{1}$ stand for $K_{0 \nu}$ and $K_{1 \nu}$ defined by Eqs. (21a) and $(21 b)$.

The solution of Eq. (36) is given by

$$
w_{0}(s)=A_{1} \sin \lambda s+A_{2} \cos \lambda s
$$

where $\lambda^{2}=\left(\Omega_{0}^{2}-1\right) / f_{0}$ has been posed. By using boundary conditions, we obtain the following characteristic equation:

$$
\tan \lambda=\frac{\beta+\gamma}{\lambda^{2}-\beta \gamma} \lambda
$$

The eigenfunctions $w_{0 j}$ verify the classical orthonormality conditions for natural systems and are independent of the choice of the constant stress value.

The first-order perturbation, Eq. (37), is solved by assuming $\boldsymbol{w}_{1 j}=\Sigma_{k} a_{j k} \boldsymbol{w}_{0 k}$. The frequency correction obtained is

$$
\Omega_{1 j}^{2}=\int_{0}^{1} f_{1}(s) w_{0 j}^{\prime 2} \mathrm{~d} s
$$

from which the natural frequencies, up to the first order, are

$$
\Omega_{j}^{2}=\Omega_{0 j}^{2}+\epsilon \Omega_{1 j}^{2}=1+\int_{0}^{1} f(s) w_{0 j}^{\prime 2} \mathrm{~d} s
$$

where use has been made of Eq. (19) and of the orthonormalization conditions. The coefficients $a_{j k}$ are furnished by

$$
a_{j k}=\frac{1}{\Omega_{j}^{2}-\Omega_{k}^{2}} \int_{0}^{1} f(s) w_{0 k}^{\prime} w_{0 j}^{\prime} \mathrm{d} s
$$

It should be noticed that, whereas eigenfunctions $w_{j}$ depend on the choice of $f_{0}, \Omega_{j}^{2}$ is independent of the constant value of the stress, which only affects the frequency approximation at order $\epsilon^{0}$.

\section{Asymptotic Analysis of the In-Plane Motion}

As already observed, the two components of the in-plane motion, $u(s, t)$ and $v(s, t)$, are coupled by means of the gyroscopic forces; a measure of the coupling is given by the coefficients $c_{p}$ and $c_{q}$ appearing in Eqs. (32). In particular, the solution depends on the nondimensional parameter $\alpha^{2}$, defined by Eqs. (6), which, in the range of technical values of the geometric and mechanical properties of the system, assumes very small values, at the most of order $\mathcal{O}\left(10^{-4}\right)$. Therefore, it is worth examining the order of magnitude of the different terms of the solution in order to point out the coupling between the components of motion and to obtain an approximate solution.

To this end, two different approaches to the problem are presented. In the first one, the asymptotic behavior of the solution for small values of $\alpha^{2}$ is studied; in the second one, the general equations of motion [Eqs. (4a) and (4b)] are solved by means of a perturbation technique in which $\alpha^{2}$ is assumed as the perturbation parameter. In both cases, for simplicity, we refer to an infinite mass of the SS $(\beta=0)$. Note that the coefficients $\gamma$ and $f(s)$ are both of order $\mathcal{O}(1)$.

Asymptotic Expansion of the Constant Stress Solution

The eigenvalue problem of in-plane motion, related to the case $\beta=0$, can be written more explicitly as

$$
\left[\begin{array}{ll}
a_{11} & a_{12} \\
a_{21} & a_{22}
\end{array}\right]\left\{\begin{array}{l}
A_{1} \\
A_{3}
\end{array}\right\}=\left\{\begin{array}{l}
0 \\
0
\end{array}\right\}
$$


where

$$
\begin{aligned}
& a_{11}=\frac{c_{p} p}{2 \alpha^{2} \omega_{0}}(p \sin p-\gamma \cos p) \\
& a_{12}=\frac{c_{q} q}{2 \alpha^{2} \omega_{0}}(q \sin q-\gamma \cos q) \\
& a_{21}=\left(1-\frac{2 c_{p}}{\omega_{0}}\right) \omega_{0}^{2} \sin p-\gamma f_{0} p \cos p \\
& a_{22}=\left(1-\frac{2 c_{q}}{\omega_{0}}\right) \omega_{0}^{2} \sin q-\gamma f_{0} q \cos q
\end{aligned}
$$

being $A_{2}=A_{4}=0$ from the boundary conditions $u(0)=v(0)$ $=0$. In Eqs. (44), the coefficients $c_{p}$ and $c_{q}$ and the spatial frequencies $p$ and $q$ are functions of $\alpha^{2}$ according to Eqs. (30), (31), and (33). By expanding $\sqrt{1+\zeta}$ in terms of $\alpha^{2}$ and substituting into Eqs. (31), we get, up to the second order

$$
\begin{gathered}
p^{2}=\frac{\omega_{0}^{2}}{f_{0}}+4 \alpha^{2} \\
q^{2}=-\alpha^{2}\left(1-\omega_{0}^{2}\right)\left(1+4 \alpha^{2} \frac{f_{0}}{\omega_{0}^{2}}\right)
\end{gathered}
$$

from which, by introducing into Eqs. (33),

$$
\begin{gathered}
c_{p}=-\frac{2 \alpha^{2} \omega_{0}^{2}}{p^{2}} \\
c_{q}=\frac{\omega_{0}}{2}\left[1+\alpha^{2} \frac{f_{0}}{\omega_{0}^{2}}\left(1-\omega_{0}^{2}\right)\right]
\end{gathered}
$$

In view of these results, it is found that the eigenvalue problem [Eq. (43)] admits the $\infty^{2}$ solutions

$$
\begin{array}{lll}
A_{1} \neq 0, & A_{3}=0, & \tan p=\gamma / p \\
A_{1}=0, & A_{3} \neq 0, & \tan q=\gamma / q
\end{array}
$$

Equations (47) show that the spatial frequencies $p$ and $q$ are equal in that they are the solution of the same characteristic equation and, furthermore, they coincide with the eigenvalues $\lambda$ of the out-of-plane motion. Therefore, it emerges from Eqs. (45a) and (38) that out-of-plane and in-plane frequencies up to order $\alpha$ verify the relation $\omega_{0}^{2}=\Omega_{0}^{2}-1$. Moreover, from Eqs. (47), it appears that the harmonics in $p$ and $q$ are uncoupled. By accounting for Eqs. (46) and (47), we find that the vibration modes [Eqs. (32)] are

$$
\begin{aligned}
& u_{0}(s)=-\frac{2 \alpha^{2} \omega_{0}^{2}}{p^{2}} A_{1} \sin p s \\
& v_{0}(s)=A_{1} \sin p s
\end{aligned}
$$

or

$$
\begin{aligned}
& u_{0}(s)=\frac{\omega_{0}}{2} A_{3} \sin q s \\
& v_{0}(s)=A_{3} \sin q s
\end{aligned}
$$

In both cases, it ensues that $u_{0}(s) / v_{0}(s)=$ const.

Equations (48) show that because of the presence of $\alpha^{2}$ in Eq. (48a) the two components of motion are weakly coupled and, besides, the transverse component prevails over the axial component. For this reason, we shall refer to Eqs. (48) as prevalently transverse eigenfunctions. Because of Eq. (45a), the first vibration modes associated with values of $p$ of order $\mathcal{O}(1)$ correspond to frequencies $\omega_{0}$ of the same order.
From Eqs. (49), it ensues that $u_{0}(s) / v_{0}(s)=\omega_{0} / 2$ and, therefore, the longitudinal component of the motion is dominant with respect to the transverse one since frequencies are of order $\Theta\left(\alpha^{-1}\right)$. This is seen from Eq. (45b) if one considers that the first $q$ is of order $O(1)$. Consequently, these modal shapes will be referred to as prevalently longitudinal eigenfunctions, which are characterized by higher values of the frequencies in comparison with the prevalently transverse ones associated with the same values of $p$.

In conclusion, in both cases the coupling between the two components of motion due to the Coriolis forces is rather weak.

\section{Asymptotic Solution of the General Equations of Motion}

Let us consider the linearized equations of the in-plane motion [Eqs. (4a) and (4b)] and the corresponding boundary conditions [Eqs. (5a-5d)] where $\beta=0$.

An asymptotic solution for $\alpha^{2} \rightarrow 0$ is sought. To this end, attention is concentrated on modal shape wavelengths of order $\mathcal{O}(1)$ for which $u "=\mathcal{O}(u)$ and $v^{\prime \prime}=\mathcal{O}(v)$ and a distinction is made between two cases according to the value of $\omega$ :

1) $\omega=\mathcal{O}(1)$ : In this case, frequencies are of the same order as those of the transverse oscillations of a string. $\ddot{u}=\mathcal{O}(u)$, $\dot{u}=\mathcal{O}(u)$, and similarly for $v$.

2) $\omega=\mathcal{O}\left(\alpha^{-1}\right)$ : In this case, frequencies are of the same order as those of the longitudinal oscillations of a string. $\ddot{u}=\mathcal{O}\left(\alpha^{-2} u\right), \dot{u}=\mathcal{O}\left(\alpha^{-1} u\right)$, and similarly for $v$.

The two cases are treated separately.

\section{Transverse Type Modes}

By performing a series expansion in terms of the perturbation parameter $\alpha^{2}$, we may write the solution of Eqs. (4a) and (4b) as

$$
\begin{gathered}
u=u_{0}+\alpha^{2} u_{1} \\
v=v_{0}+\alpha^{2} v_{1}
\end{gathered}
$$

from which the perturbation equations with relevant boundary conditions are derived:

Order $\alpha^{0}$ :

$$
\begin{gathered}
u_{0}^{\prime \prime}=0 \\
u_{0}(0, t)=0 \\
u_{0}^{\prime}(1, t)=0
\end{gathered}
$$

Order $\alpha^{2}$ :

$$
\begin{gathered}
-2 \dot{v}_{0}-u_{1}^{\prime \prime}=0 \\
\ddot{v}_{0}-\left[f(s) v_{0}^{\prime}\right]^{\prime}=0 \\
u_{1}(0, t)=0 \\
v_{0}(0, t)=0 \\
-2 \dot{v}_{0}(1, t)+\gamma u_{1}^{\prime}(1, t)=0 \\
\ddot{v}_{0}(1, t)+\gamma f(1) v_{0}^{\prime}(1, t)=0
\end{gathered}
$$

The solution of Eqs. (51) and (52) furnishes $u_{0}(s, t)=0$ and, therefore, $u=\mathcal{O}\left(\alpha^{2}\right)$. Equation (53b) describes the vibrations of a string under variable tension $f(s)$ and admits a solution of the type

$$
v_{0}(s, t)=v_{0}(s) \cos (\omega t+\psi)
$$

where $v_{0}(s)$ and $\omega$ have to be determined by imposing the boundary conditions [Eqs. (54b) and (54d)]. If $f(s)$ is approx- 
imated with a constant value $f_{0}$, for instance, its average value, the solution is given by $v_{0}(s)=A$ sinps. The associated value of the frequencies is

$$
\omega_{0}=\sqrt{f_{0}} p
$$

where $p$ is the solution of the characteristic equation

$$
\tan p=\gamma / p
$$

By performing integrations on Eq. (53a) and by using the characteristic equation and appropriate boundary conditions, we obtain $u(s, t)$. Therefore, at the present level of the perturbation procedure, the solution reads

$$
\begin{gathered}
u(s, t)=-2 \alpha^{2} \frac{\omega_{0}}{p^{2}} A \sin p s \sin \left(\omega_{0} t+\psi\right) \\
v(s, t)=A \sin p s \cos \left(\omega_{0} t+\psi\right)
\end{gathered}
$$

Within this approximation, the following results have been achieved.

1) The frequency coincides with that of the transverse oscillations of a string and it is not modified by the longitudinal motion.

2) The amplitude of the longitudinal displacement $u(s, t)$ is small of order $\mathcal{O}\left(\alpha^{2}\right)$ in comparison with the transverse one.

3) The displacement components $u(s, t)$ and $v(s, t)$ oscillate with a phase difference of $\pi / 2$.

Note that the frequencies and the modal shapes coincide with those given by the series expansion of the exact solution of the coupled oscillations with constant stress.

The employed perturbation procedure is susceptible to a useful interpretation that helps understanding of the mechanical behavior of the system. The equation that governs the most important aspects of the phenomenon is Eq. (53b), which, as previously stated, corresponds to a purely transverse motion. Equation (53a) represents the equilibrium condition in the longitudinal direction of the string where $u_{1}^{\prime \prime}$ and $\dot{v}_{0}$ are, respectively, proportional to the elastic reaction and to the longitudinal component of the Coriolis force generated by the transverse motion. A stress increment, which is proportional to the linearized stretching $u_{1}^{\prime}$, arises in the string to balance, on the section of abscissa $s$, the Coriolis' forces acting on the cable from the current section up to the free end as well as the Coriolis' force on the satellite. It can be seen that the stress increment is small in comparison with the prestress.

At the next order, which is not examined here, the longitudinal displacements appear in the transverse equilibrium equation by producing a Coriolis' force proportional to $\dot{u}_{1}$ responsible for a small frequency correction. In conclusion, the procedure shows that in the prevalently transverse motion the axial displacement of the cable represents only a secondary aspect of the phenomenon if the cable is characterized by sufficiently high axial rigidity.

\section{Longitudinal Type Modes}

In this case, it is convenient to modify the time scale by introducing a new variable $\tau=\alpha^{-1} t$. It is, therefore, $\omega t=\omega \alpha \tau=\bar{\omega} \tau$ where $\bar{\omega}=\omega \alpha=\mathcal{O}(1)$. In the new time scale, it is $\partial u(s, \tau) / \partial \tau=\mathcal{O}(\bar{\omega} u)=\mathcal{O}(u), \partial^{2} u(s, \tau) / \partial \tau^{2}=\mathcal{O}\left(\bar{\omega}^{2} u\right)=\mathcal{O}(u)$.

By expanding $u$ and $v$ in series of $\alpha$, i.e.,

$$
\begin{aligned}
& u=u_{0}+\alpha u_{1} \\
& v=v_{0}+\alpha v_{1}
\end{aligned}
$$

we obtain the relevant perturbation equations through standard steps. By solving the equations and coming back to variable $t$, we obtain

$$
u(s, t)=A \sin q s \sin (\omega t+\psi)
$$

$$
v(s, t)=(2 / \omega) A \sin q s \cos (\omega t+\psi)
$$

where $\omega^{2}=q^{2} / \alpha^{2}$ and $q$ satisfies the characteristic equation tan $q=\gamma / q$. It is interesting to observe that the characteristic equation coincides with Eqs. (47b), Eqs. (59) are in accordance with Eqs. (49), and, besides, $\omega^{2}$ is a good approximation of Eq. (45b) since $\omega \gg 1$. Hence, in this case too, the solution previously obtained is recovered.

\section{Numerical Results}

The main results presented here regard the analysis of a TSS with $m_{1} \gg m_{2}$; some results relative to systems with equal concentrated masses will be illustrated at the end of the section. Let us assume the orbital angular velocity $n^{2}=$ $1.35 \cdot 10^{-6} \mathrm{~s}^{-2}$, orbital radius $a=6657 \mathrm{~km}$, SS mass $m_{1}=10^{5}$ $\mathrm{kg}$, satellite mass $m_{2}=500 \mathrm{~kg}$, tether mass $\mu=5.76 \cdot 10^{-3} \mathrm{~kg} /$ $\mathrm{m}$, and tether cross section $A=4 \cdot 10^{-6} \mathrm{~m}^{2}$, Young's modulus $E=7 \cdot 10^{10} \mathrm{~N} / \mathrm{m}^{2}$, and tether length $\ell$ is a variable. Two systems are analyzed in detail corresponding to $\ell=20$ and $100 \mathrm{~km}$, for which nondimensional parameters assume the values $\alpha^{2}=1.111 \cdot 10^{-5}, \beta=0.001152, \gamma=0.2304, \delta=0.0055$, and $\alpha^{2}=2.777 \cdot 10^{-4}, \beta=0.00576, \gamma=1.152, \delta=0.0078$, respectively. The analysis has been performed by referring to the solution reported in the third section and results are compared with those of the asymptotic solution.

Table 1 collects the in-plane frequencies for $\ell=100 \mathrm{~km}$ corresponding to three values of $f_{0}$, namely, the lowest $\left(f_{\min }=2.585\right)$, the average $\left(f_{\mathrm{av}}=3.574\right)$, and the largest $\left(f_{\max }=4.062\right)$ value of $f(s)$. It is noticed that, whereas $\omega_{0}$ usually grows with $f_{0}$, the values of $\omega=\omega_{0}+\epsilon \omega_{1}$ are practically independent of $f_{0}$. Besides, the corrections $\omega_{1}$ relative to the average value of $f(s)$ are very small and decrease with the mode number, thus giving $\omega \cong \omega_{0}$. Similar results are obtained for a length of $20 \mathrm{~km} .{ }^{15}$

A hypothesis that permits simplification of the numerical analysis consists in assuming that the SS mass is infinite, which implies $\beta=0$. Under this assumption, apart from the first modes, results change very little. For decreasing lengths, the difference between results corresponding to $\beta=0$ and $\beta \neq 0$ tends to disappear.

Numerical results, confirmed by the asymptotic analysis, show that the vibration modes are characterized by a weak coupling between the two components of motion $u_{0}(s)$ and $v_{0}(s)$, and, therefore, they are of prevalently transversal or longitudinal type. In particular, among the first modes, most are of transversal type except for a few, for instance, $i=11$, $37, \ldots$ for $\ell=100 \mathrm{~km}$ or $i=13, \ldots$ for $\ell=20 \mathrm{~km}$. The corre sponding frequencies $\omega_{0}$ grow with $f_{0}$ in the prevalently transversal modes since they are related to the geometric stiffness or they remain practically constant in the prevalently longitudinal ones because of their dependence on EA (see

\begin{tabular}{|c|c|c|c|c|c|c|}
\hline \multirow[b]{2}{*}{ Mode } & \multicolumn{2}{|c|}{$f_{\min }$} & \multicolumn{2}{|c|}{$f_{\mathrm{av}}$} & \multicolumn{2}{|c|}{$f_{\max }$} \\
\hline & $\omega_{0}$ & $\omega$ & $\omega_{0}$ & $\omega$ & $\omega_{0}$ & $\omega$ \\
\hline 1 & 1.467 & 1.819 & 1.725 & 1.794 & 1.840 & 1.797 \\
\hline 2 & 5.590 & 7.046 & 6.577 & 6.905 & 7.018 & 6.900 \\
\hline 3 & 10.426 & 12.672 & 12.265 & 12.475 & 13.088 & 12.488 \\
\hline 4 & 15.404 & 18.534 & 18.122 & 18.269 & 19.338 & 18.299 \\
\hline 5 & 20.426 & 24.480 & 24.030 & 24.143 & 25.643 & 24.187 \\
\hline 11 & 54.559 & 54.559 & 54.559 & 54.555 & 54.559 & 54.559 \\
\hline 37 & 207.883 & 207.883 & 207.883 & 207.883 & 207.883 & 207.883 \\
\hline
\end{tabular}
Table 1). It is found that harmonics with circular frequency $p$ dominate over those in $q$ in the prevalently transversal modes, whereas the opposite occurs in the prevalently longitudinal modes. This has been pointed out by the asymptotic analysis according to which transverse modes contain only harmonics in $p$ [Eqs. (48) and (57)] and longitudinal modes contain only

Table 1 Time frequencies of in-plane oscillations $(\ell=100 \mathrm{~km})$ 
those in $q$ [Eqs. (49) and (59)]. From this, it follows that the motion components $u_{0}(s)$ and $v_{0}(s)$ can be assumed proportional, with constant ratio equal to $c_{p}$ and $c_{q}$, respectively.

Figures 2 show plots of the transversal and longitudinal displacements relative to mode numbers $1-4,11$, and 37 for $\ell=100 \mathrm{~km}$ and mode numbers $1-5$ and 13 for $\ell=20 \mathrm{~km}$, evaluated for $f_{0}=f_{\mathrm{av}}$ in both cases. The values of the ratio $u_{0} / v_{0}$ reported in Figs. 2 confirm that the motion components are practically uncoupled. The first modes in Figs. $2 a$ and $2 b$ are of a pendular type, whereas the subsequent modes are similar to those of a vibrating string, except for the presence of a small displacement at the end $s=1$ where the satellite is attached; also at $s=0$ a small displacement of the SS occurs, especially for great lengths of the tether. The behavior of $u_{0}(s)$ at prevalently longitudinal modes is similar to that of $v_{0}(s)$ at corresponding prevalently transversal ones, although $\omega_{0}$ is much higher.

The values of $p$ and $q$ are collected in Table 2 for $\ell=100 \mathrm{~km}$. Each frequency $p$ of the prevalently transverse modes is matched by one frequency $q$ of the prevalently longitudinal modes, and, therefore, the wavelengths of the two modes are approximately the same. These spatial frequencies are approximately multiples of $\pi$, except for the first modes. It is found that these values are independent of the choice of the constant value $f_{0}$, according to Eqs. (47). Besides, they are practically the same as frequencies $\lambda$ of out-of-plane modes, also listed in Table 2.

Previous results regarding the weak coupling between $u_{0}(s)$ and $v_{0}(s)$ are confirmed by evaluating the kinetic and potential
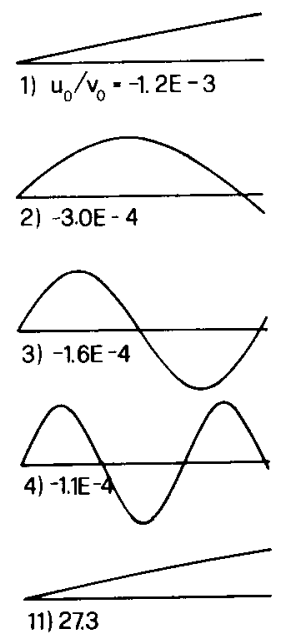

a)

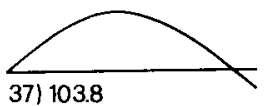

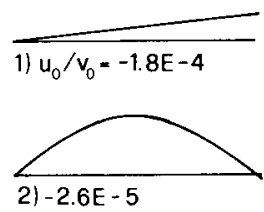
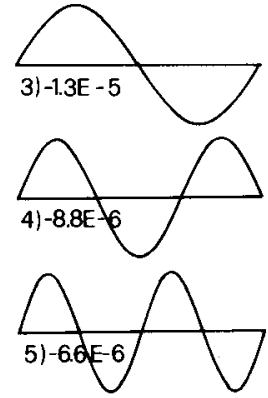

b)

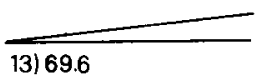

Fig. 2 In-plane modal shapes: a) $\ell=100 \mathrm{~km}$; b) $\ell=20 \mathrm{~km}$.

Table 2 Time and spatial frequencies of in-plane and outof-plane oscillations $\left(f_{0}=f_{\mathrm{av}}, \ell=100 \mathrm{~km}\right)$

\begin{tabular}{|c|c|c|c|c|c|}
\hline \multirow[b]{2}{*}{ Mode } & \multicolumn{3}{|c|}{ In-plane oscillations } & \multicolumn{2}{|c|}{ Out-of-plane oscillations } \\
\hline & $\omega$ & $p / \pi$ & $q / \pi$ & $\Omega$ & $\lambda / \pi$ \\
\hline 1 & 1.794 & 0.289 & 0.007 & 2.006 & 0.289 \\
\hline 2 & 6.905 & 1.103 & 0.034 & 6.587 & 1.103 \\
\hline 3 & 12.457 & 2.056 & 0.065 & 12.263 & 2.056 \\
\hline 4 & 18.269 & 3.038 & 0.096 & 18.119 & 3.038 \\
\hline 5 & 24.143 & 4.029 & 0.127 & 24.027 & 4.029 \\
\hline 10 & 53.807 & 9.013 & 0.285 & 53.754 & 9.013 \\
\hline 11 & 54.559 & 9.148 & 0.289 & 59.724 & 10.012 \\
\hline 12 & 59.758 & 10.012 & 0.317 & 65.680 & 10.823 \\
\hline 37 & 207.883 & 34.855 & 1.103 & 214.734 & 36.003 \\
\hline
\end{tabular}

energy terms present in Eq. (24). Table 3 shows that the energy terms relative to $u_{0}(s)$ are small in comparison with those relative to $v_{0}(s)$ in the prevalently transverse modes, whereas they become dominant in the prevalently longitudinal modes.

Figure 3 shows a plot of the time frequencies vs the mode number for different lengths of the tether. It is apparent that, for each value of the length, the behavior is practically linear, with the exception of the first modes; besides, the time frequencies tend to be independent of the length as it increases. This is confirmed by considering the perturbation equation [Eq. (53b)] (no gyroscopic forces) and Eq. (8). It is seen that for $\ell \rightarrow \infty, f(s) \rightarrow 3\left(s-s^{2}\right) / 2$, i.e., the tension is length independent; consequently, time frequencies tend to be a limit value different from zero.

By enlarging the abscissa scale, we notice that the original straight line associated with each value of the length translates to parallel paths by correspondence with mode numbers $i=11,37, \ldots$ for $\ell=100 \mathrm{~km}$ or $i=13, \ldots$ for $\ell=20 \mathrm{~km}$; these jumps are associated with prevalently longitudinal modes. Figure 4 and Table 2 illustrate this situation together with the behavior of out-of-plane time frequencies $\Omega$ (straight lines in Fig. 4). Note that the amount of the translation, measured along the horizontal line, is equal to one mode and the same difference occurs between in-plane and out-of-plane spatial frequencies.

The coefficients $a_{j k}$ relative to the shape corrections of the eigenfunctions of in-plane vibrations have been determined by solving the appropriate system of equations. It is found that these equations are practically uncoupled since the ratio between out-of-diagonal and diagonal coefficients is at the most of order $\mathcal{O}\left(10^{-3}\right)$, because the wavelengths of the eigenfunctions are approximately multiples of $\pi$. Table 4 shows the values of $a_{j k}$ calculated by considering for $f_{0}$, the average value of $f(s)$. It is apparent that these values rapidly decrease starting from the mode to be corrected so that, in the analysis, it is sufficient to consider a narrow band around the mode in question. Similar results are obtained for out-of-plane motion (see Ref. 15).

Results obtained so far are compared with those illustrated in Ref. 10. The present solution depends on three nondimen-

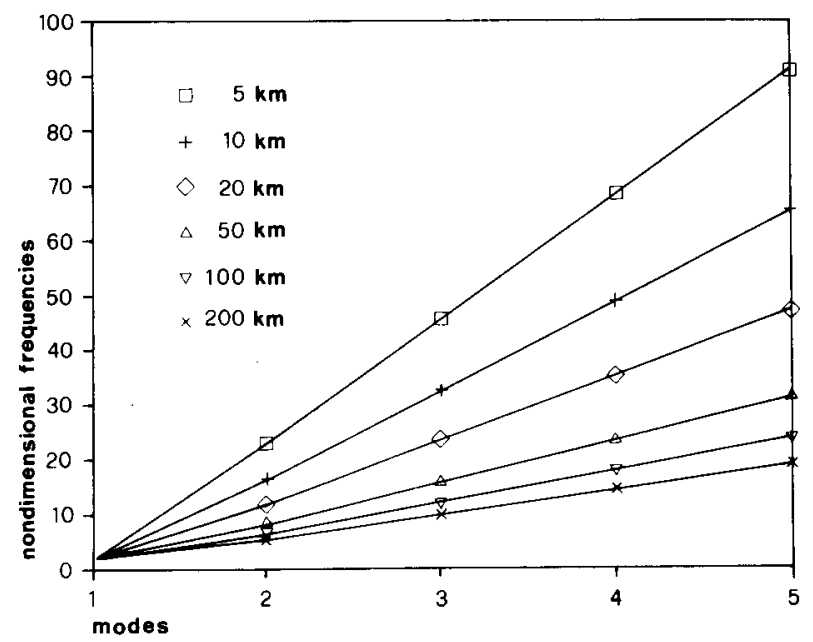

Fig. 3 In-plane time frequencies for different lengths of the tether.

Table 3 Energy contributions of $u_{0}$ and $v_{0}(\ell=100 \mathrm{~km})$

\begin{tabular}{clccc}
\hline \hline Mode & Kinetic $u_{0}$ & Potential $u_{0}$ & Kinetic $v_{0}$ & Potential $v_{0}$ \\
\hline 1 & $3.91 \mathrm{E}-7$ & $1.16 \mathrm{E}-3$ & $2.90 \mathrm{E}-1$ & $8.62 \mathrm{E}-1$ \\
2 & $7.06 \mathrm{E}-9$ & $3.05 \mathrm{E}-4$ & $7.60 \mathrm{E}-2$ & $3.29 \mathrm{E}+0$ \\
3 & $1.09 \mathrm{E}-9$ & $1.64 \mathrm{E}-4$ & $4.08 \mathrm{E}-2$ & $6.13 \mathrm{E}+0$ \\
4 & $3.37 \mathrm{E}-10$ & $1.11 \mathrm{E}-4$ & $2.76 \mathrm{E}-2$ & $9.06 \mathrm{E}+0$ \\
5 & $1.46 \mathrm{E}-10$ & $8.35 \mathrm{E}-5$ & $2.08 \mathrm{E}-2$ & $1.20 \mathrm{E}+1$ \\
11 & $9.16 \mathrm{E}-3$ & $2.72 \mathrm{E}+1$ & $1.23 \mathrm{E}-5$ & $3.68 \mathrm{E}-5$ \\
37 & $2.41 \mathrm{E}-3$ & $1.04 \mathrm{E}+2$ & $2.23 \mathrm{E}-7$ & $9.65 \mathrm{E}-6$ \\
\hline \hline
\end{tabular}


Table 4 Coefficients $a_{j k}$ of the shape corrections of in-plane modes $(\ell=100 \mathrm{~km})$

\begin{tabular}{ccccccc}
\hline \hline Mode & $j-3$ & $j-2$ & $j-1$ & $j+1$ & $j+2$ & $j+3$ \\
\hline 1 & - & - & & $-6.56 \mathrm{E}-2$ & $9.44 \mathrm{E}-3$ & $-3.21 \mathrm{E}-3$ \\
2 & - & $-\overline{6.81 \mathrm{E}}-4$ & $4.66 \mathrm{E}-2$ & $-1.36 \mathrm{E}-1$ & $2.30 \mathrm{E}-2$ & $-8.55 \mathrm{E}-3$ \\
3 & - & $-8.26 \mathrm{E}-3$ & $9.06 \mathrm{E}-2$ & $-1.76 \mathrm{E}-1$ & $2.78 \mathrm{E}-2$ & $-1.00 \mathrm{E}-2$ \\
4 & $2.86 \mathrm{E}-4$ & $-5.26 \mathrm{E}-3$ & $1.32 \mathrm{E}-1$ & $-2.17 \mathrm{E}-1$ & $3.28 \mathrm{E}-2$ & $-1.15 \mathrm{E}-2$ \\
5 & $1.47 \mathrm{E}-3$ & $-1.13 \mathrm{E}-2$ & $1.32 \mathrm{E}-1$ & \\
\hline
\end{tabular}

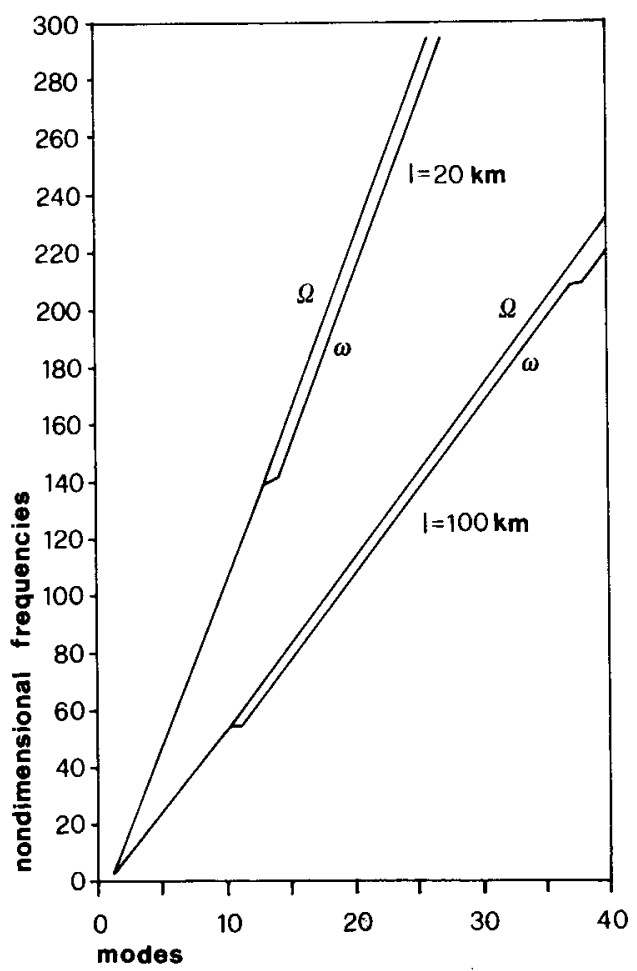

Table 5 In-plane and out-of-plane frequencies $\left(\alpha^{2}=1.111 \cdot 10^{-5}, \beta=0, \gamma=0.2\right)$

\begin{tabular}{|c|c|c|c|c|c|c|}
\hline \multirow[b]{2}{*}{ Mode } & \multicolumn{4}{|c|}{ Present theory } & \multicolumn{2}{|c|}{ Reference 10} \\
\hline & $\omega_{0}$ & $\omega$ & $\Omega_{0}$ & $\Omega$ & $\omega$ & $\mathbf{\Omega}$ \\
\hline 1 & 1.731 & 173 & 1.999 & 2.000 & 1.732 & 2.0 \\
\hline 2 & 12.816 & 12.780 & 12.854 & 12.819 & 12. & \\
\hline 3 & 25.259 & 25.241 & 25.279 & 25. & 25.174 & \\
\hline 4 & 37.784 & 37.771 & 37.797 & & & \\
\hline 5 & 50.329 & 50.319 & 50.339 & 50.329 & 50.305 & 50. \\
\hline
\end{tabular}

Table 6 In-plane and out-of-plane frequencies $\left(\alpha^{2}=1.111 \cdot 10^{-5}, \beta=0, \gamma=1\right)$

\begin{tabular}{|c|c|c|c|c|c|c|}
\hline \multirow[b]{2}{*}{ Mode } & \multicolumn{4}{|c|}{ Present theory } & \multicolumn{2}{|c|}{ Reference 10} \\
\hline & $\omega_{0}$ & $\omega$ & $\Omega_{0}$ & $\Omega$ & $\omega$ & $\mathbf{\Omega}$ \\
\hline 1 & 1.721 & 1.742 & 1.990 & 2.000 & 1.732 & 2.000 \\
\hline 2 & 6.851 & 6.750 & 6.294 & 6.823 & 6.707 & 6.782 \\
\hline 3 & 12.875 & 12.811 & 12.913 & 12.850 & 12.742 & 12.782 \\
\hline 4 & 19.059 & 19.014 & 19.085 & 19.040 & 18.915 & 18.942 \\
\hline 5 & 25.291 & 25.256 & 25.310 & 25.276 & 25.127 & 25.147 \\
\hline
\end{tabular}

Fig. 4 Time frequencies of in-plane and out-of-plane oscillations.

Table 7 In-plane frequencies and displacements $(\ell=20 \mathrm{~km})$

\begin{tabular}{|c|c|c|c|c|c|c|c|}
\hline \multirow[b]{2}{*}{ Mode } & \multicolumn{3}{|c|}{$\beta=0.001152, \gamma=0.23$} & \multicolumn{2}{|c|}{$\beta=\gamma=0.001152$} & \multicolumn{2}{|c|}{$\beta=\gamma=0.2304$} \\
\hline & $\omega$ & $\left|v_{1} / v_{\max }\right|$ & $\left|v_{2} / v_{\max }\right|$ & $\omega$ & $\left|v_{1,2} / v_{\max }\right|$ & $\omega$ & $\left|v_{1,2} / v_{\max }\right|$ \\
\hline 1 & 1.738 & $5.58 \mathrm{E}-3$ & 1.0 & 1.728 & 1.0 & 1.742 & 1.0 \\
\hline 2 & 11.993 & $3.58 \mathrm{E}-4$ & $7.15 \mathrm{E}-2$ & 113.385 & $3.66 \mathrm{E}-4$ & 8.341 & $7.00 \mathrm{E}-2$ \\
\hline 3 & 23.644 & $1.82 \mathrm{E}-4$ & $3.64 \mathrm{E}-2$ & 226.736 & $1.83 \mathrm{E}-4$ & 16.191 & $3.62 \mathrm{E}-2$ \\
\hline 4 & 35.368 & $1.21 \mathrm{E}-4$ & $2.43 \mathrm{E}-2$ & 340.095 & $1.22 \mathrm{E}-4$ & 24.155 & $2.43 \mathrm{E}-2$ \\
\hline 5 & 47.112 & $0.92 \mathrm{E}-4$ & $1.83 \mathrm{E}-2$ & 453.455 & $0.92 E-4$ & 32.142 & $1.82 \mathrm{E}-2$ \\
\hline
\end{tabular}

sional parameters $\alpha^{2}, \beta, \gamma$ [Eqs. (6)], whereas the solution in Ref. 10 depends on the unique parameter $\gamma$. This is because, in were neglected, thus implying the transversal modes to be independent of $\alpha^{2}$. On the other hand, the solution in Ref. 10 is more accurate than the present one since Legendre polynomials are employed. Tables 5 and 6 collect the first five inplane $\omega$ and out-of-plane $\Omega$ frequencies of two systems with $\alpha^{2}=1.111 \cdot 10^{-5}, \beta=0$, and $\gamma=0.2$ and 1 , respectively. When $\gamma=0.2$, it is seen that even zeroth-order frequencies $\omega_{0}$ and $\Omega_{0}$ are in good accord with result of Ref. 10; the agreement is far better for first-order frequencies. When $\gamma=1$, the approximation is not as good, but still acceptable. For increasing $\gamma$, differences increase because the stress $f(s)$ is no more slowly variable, and, therefore, the hypothesis of applicability of the perturbation method fails.

Finally, the influence of the lumped masses $m_{1}$ and $m_{2}$ on the frequencies and modal shapes of the system are analyzed. Three TSS are considered and results relative to the in-plane transversal modes are compared. The first system is one of those previously considered, with $m_{1}=10^{5} \mathrm{~kg}, m_{2}=500 \mathrm{~kg}$, whose modal shapes are plotted in Fig. $2 \mathrm{~b}$; in the second system, $m_{1}=m_{2}=10^{5} \mathrm{~kg}$, in the third one, $m_{1}=m_{2}=500 \mathrm{~kg}$. The mass of the tether is the same in the three cases. The first-order frequencies $\omega$ displayed in Table 7 are seen to be strongly dependent on the increase of the masses because of the increasing of the stress, except for the pendular mode whose frequency is stress independent. The ratios between the two end displacements $v_{1}$ and $v_{2}$ and the maximum displacement $v_{\max }$ are also shown in the table. It is seen that, starting from the second mode, when the masses are both large, the modal shapes are nearly sinusoidal with zero displacement at the ends; when the masses decrease, the displacement becomes noticeable. It is interesting to note that the displacement at one end is nearly independent of the value of the mass at the other end, especially for the higher modes. Finally, the first mode of the TSS with equal masses is an approximation of the exact 